\title{
Negative phototaxis in M. incognita
}

\author{
Morihiro Oota ${ }^{1}$, Eiji Gotoh ${ }^{2}$, Masaharu Endo ${ }^{1}$, Takashi Ishida ${ }^{1}$, Tomonao Matsushita ${ }^{2,3}$, \& Shinichiro Sawa ${ }^{1}$ \\ ${ }^{1}$ Graduate School of Science and Technology, Kumamoto University, Kumamoto 860-8555, Japan \\ ${ }^{2}$ Faculty of Agriculture, Kyushu University, Fukuoka 812-8581, Japan \\ ${ }^{3}$ PRESTO, JST, Saitama 332-0012, Japan
}

Correspondence: Shinichiro Sawa, Graduate School of Science and Technology, Kumamoto University, Kumamoto 860-8555, Japan. E-mail: sawa@kumamoto-u.ac.jp

Received: May 2, 2017

doi:10.5539/ijb.v9n3p51
Accepted: May 18, 2017

Online Published: May 22, 2017

\begin{abstract}
Phototaxis is a well-known behavior in many animals. However, little is known whether nematodes living in soil recognize light. Meloidogyne incognita is one of the most important root-knot nematode pests in the world. It has a very wide host range and can infect almost all plant species. Here, we examined the phototactic response in $M$. incognita. Negative phototactic behavior was observed in M. incognita on an agar plate. Nematodes responded by avoiding white light and blue light more than red light on agar plates. Light avoidance behavior of the nematodes was examined by microscopic analysis, and the nematodes escaped from near ultraviolet light (365 $\mathrm{nm})$ in only approximately 3.2 seconds. They also avoided blue $(470 \mathrm{~nm})$, blue-green $(500 \mathrm{~nm})$, and green $(550$ $\mathrm{nm}$ ) light in approximately 4.1, 7.4, and 10.0 seconds, respectively. These results suggest that $M$. incognita exhibits negative phototaxis, in particular, responding efficiently to shorter wavelength of light.
\end{abstract}

Keywords: M. incognita, nematode, root knot, negative phototaxis

\section{Introduction}

Phototaxis is a common behavior in many animals. In many cases, phototaxis is important for animal survival. However, animals living in darkness sometimes lack the ability to respond to light. Approximately $50 \%$ of marine nematodes are thought to have photoreceptors (McLaren, 1973). Oncholaimus vesicarius and Enoplus anisospiculus are reported to have negative phototaxis to blue light (Burr., 1979). However, it is not well known whether M. incognita living in soil recognize light.

The nematode $C$. elegans is reported to possess light-sensitive neurons with CNG channel-mediated phototaxis (Cho et al., 2005; Ward et al., 2008). Furthermore, some marine and freshwater nematodes, O. vesicarius and $E$. anisospiculus contain pigments in their heads and are phototactic, though no molecular data are available because of limited genome information (Chitwood \& Murphy, 1964; Croll, 1966; Burr, 1979).

One of the major nematode pests, $M$. incognita, causes great damage to agricultural production and lives in the soil without light. However, whether M. incognita possesses phototaxis or pigments remains unknown.

Here, we showed that, despite the lack of specialized light-sensing organs in the head or other parts of the body, $M$. incognita exhibits negative phototactic behavior that is efficiently induced by shorter wavelength of light. This behavior seems to be important for survival to avoid toxic ultraviolet light, and it may contribute to the nematode's ability to live in soil without light.

\section{Materials and Methods}

\subsection{Nematode Preparation}

M. incognita were prepared as in Nishiyama et al. (2015). Briefly, five or six tomato plants (cultivar Pritz; Kaneko Seeds Co., Ltd., Japan) were inoculated with approximately 120,000 M. incognita J2 in soil. The nematodes were isolated from Koshi, Kumamoto, Japan, and maintained in the NARO Kyushu Okinawa Agricultural Research Center. Two weeks after the inoculation, the tomatoes were moved to a hydroponic culture system, and nematodes were collected from the liquid medium. 


\subsection{Long-Term Phototaxis Assay on Agar Plates}

$1.5 \%$ agar medium without any nutrients was prepared in plastic petri dishes $6 \mathrm{~cm}$ in diameter (TPP Ltd., Japan). A total of 2,000 nematodes were placed in the center of an agar plate and left for $12 \mathrm{~h}$ at room temperature with a black paper shade (Figure 1A). Agar plates were irradiated by white light (fluorescent lamp, EFD15EN/13), blue light (470 nm LED, IS-mini, CCS, Japan), and red light (660 nm LED, IS-mini, CCS, Japan) from one side (Figure 1A). The distribution of nematodes on each agar plate was observed and photographed by a GT-F600 scanner (Epson). Binarization of nematode distributions was performed using Image J v1.46r (NIH, USA) (Figure 1B). Three strips, each $3 \mathrm{~cm}$ wide, were appointed in the binarized photograph, and integration of the nematode area was calculated by Image J (Figure 1B). An average of the integrated data for the three strips was calculated by Microsoft Excel, version 15.16.
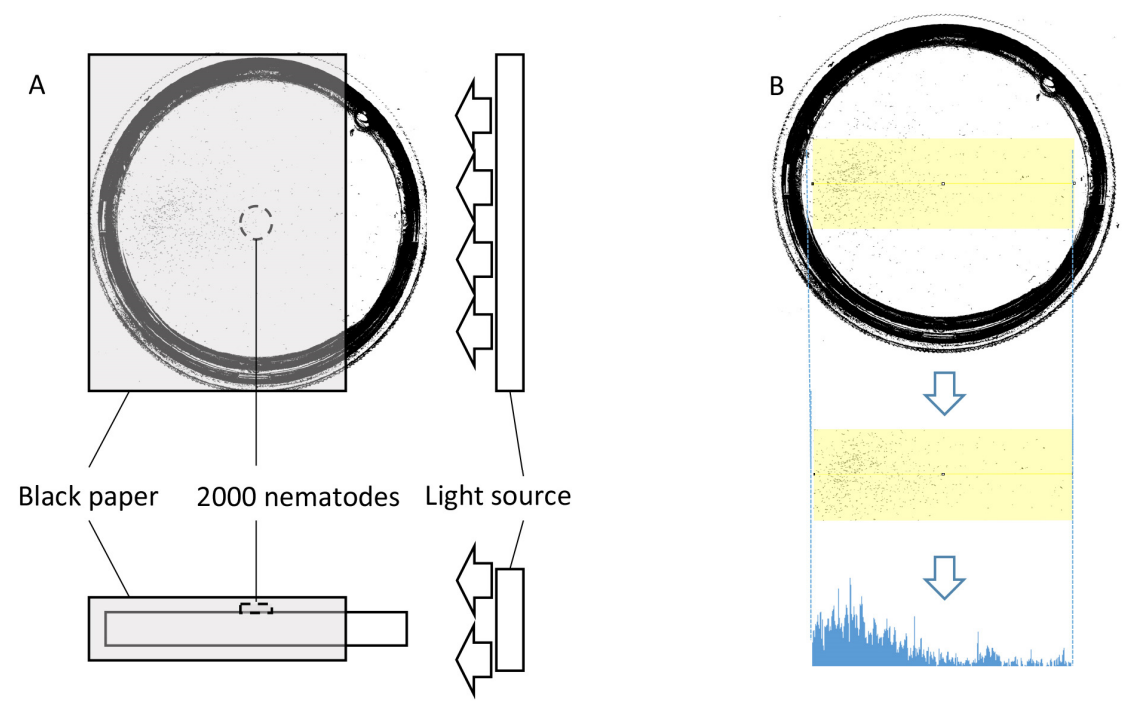

Figure 1. Methods for evaluating nematode phototaxis. A: Nematodes were placed in the center of a $1.5 \%$ agar plate (dotted circle) and left for $12 \mathrm{~h}$ with $80 \%$ of the plate shaded by black paper. Light sources were placed 5 $\mathrm{cm}$ away from the plate. B: Calculation of nematode distribution. The photographs were binarized by Image J. A stripe area ( $3 \mathrm{~cm}$ wide; first arrow) was sampled from the nematode plate. The black dotted area was considered the nematode area and was integrated at each point from left to right (second arrow)

\subsection{Short Term Phototaxis Assay Using Short-Wavelength Light}

Nematodes were irradiated by ultraviolet ( $365 \mathrm{~nm}$ : filter set 49 , Zeiss), blue (470 $\mathrm{nm}$ : filter set $38 \mathrm{HE}$, Zeiss), blue-green $(500 \mathrm{~nm}$ : filter set 46, Zeiss), and green (550 nm: filter set 43HE, Zeiss) lights on a glass slide, observed using an upright microscope (Imager M1, Zeiss, Germany), and photographed in animation using a camera (DP71, Olympus). Light avoidance duration was measured using a soft, DP 3.2, Olympus.

\section{Results}

Long-term phototaxis assay on agar plates was performed using $M$. incognita by irradiating white, blue and red light. Most of all nematodes were observed on the side of the agar plate opposite to the white-light irradiation (Figure 2A). The nematode density was higher on the side opposite the light source. We further examined blue light (short-wavelength light) and red light (long-wavelength light). We found that blue light triggered negative phototaxis more efficiently than red light in nematodes (Figure 2B, C). 

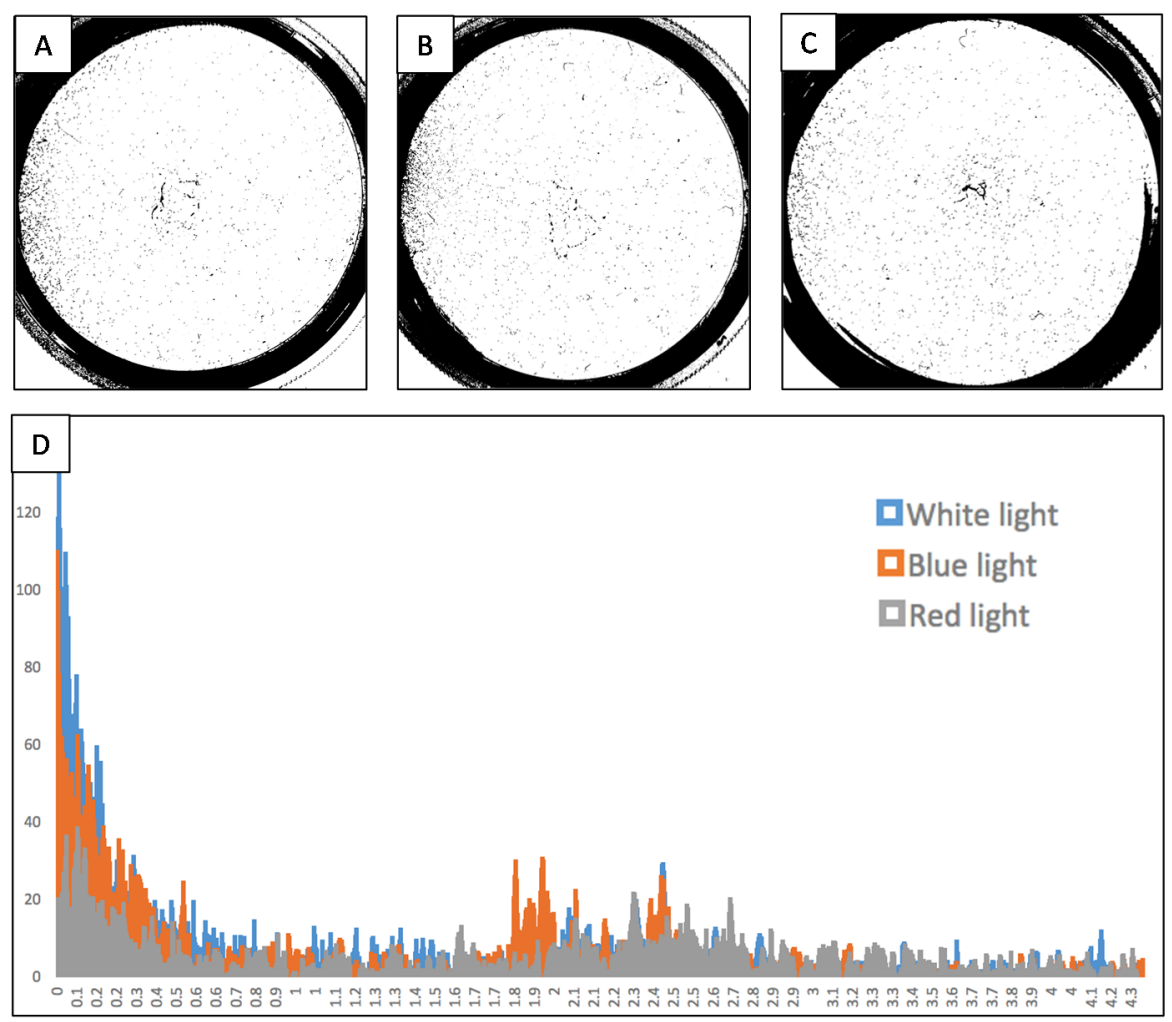

Figure 2. Long term phototaxis assay of M. incognita. Distribution of nematodes irradiated by white light (A), blue light (B), and red light (C) (n=3). D: Nematode distributions expressed as integrations of the black areas. The $\mathrm{x}$-axis gives the distance $(\mathrm{cm})$ from the left side of the stripe as shown in Figure 1B. The y-axis shows the integration area of the black pixels

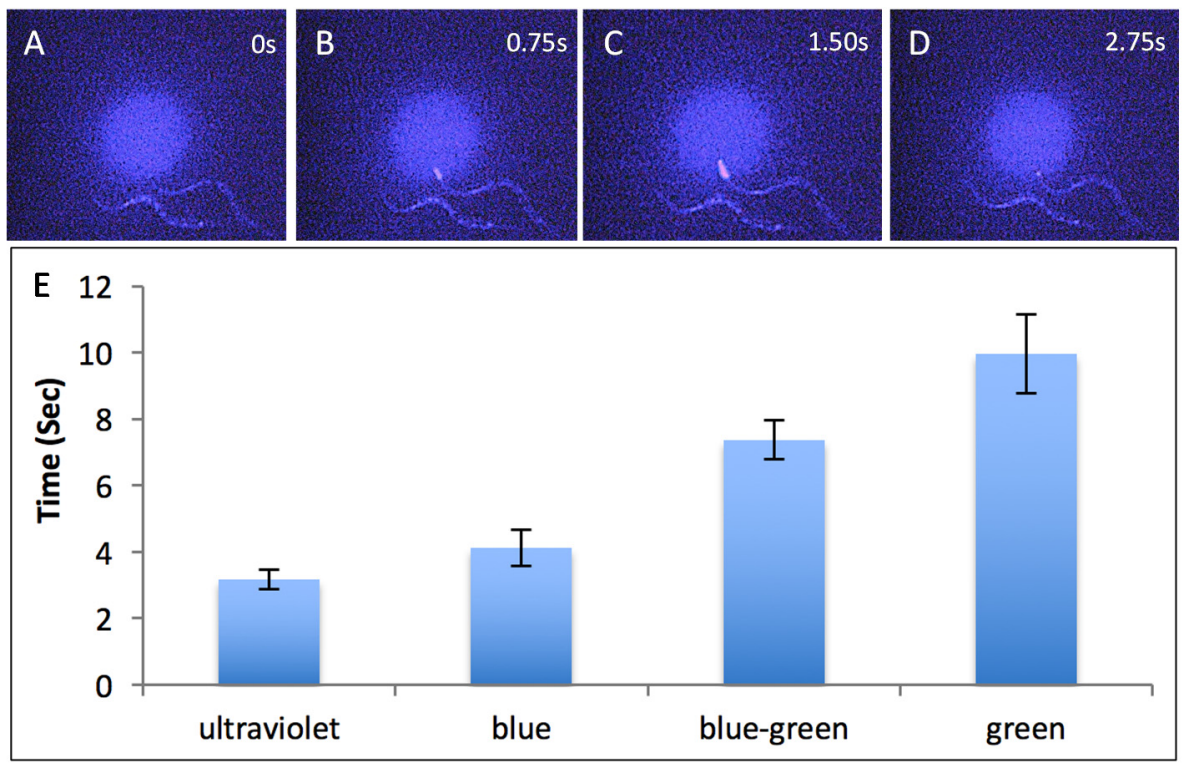

Figure 3. Short-term phototaxis assay of $M$. incognita. Snapshot images showing ultraviolet light avoidance responses in nematodes moving forward (A-D). Light was delivered via the objective to the head of a worm moving forward under the microscope. In this case, the nematode responded by stopping forward movement at $1.5 \mathrm{~s}$ and initiating reversal, leaving the light irradiation area at $2.75 \mathrm{~s}$. E: The average time for head avoidance responses for ultraviolet, blue, blue-green, and green light. The data are the means with the error bars indicating the standard deviations $(n=42)$ 
The negative phototaxis results were also expressed as a histogram. The shaded area of nematodes in the $3-\mathrm{cm}$ strips on the agar plate was integrated, as shown in Figure 1B. The nematodes were significantly distributed toward the side opposite the white- and blue-light irradiation. Red light led to a limited negative phototactic response (Figure 2D). These results indicate that $M$. incognita negatively responds to white and blue light more than to red light.

Because blue light was more effective than red light at inducing a response (Figure 2B-D), the short-term response of nematodes to shorter wavelength of light was examined (Figure 3). Nematodes were independently irradiated by near ultraviolet $(365 \mathrm{~nm})$, blue $(470 \mathrm{~nm})$, blue-green $(500 \mathrm{~nm})$, or green $(550 \mathrm{~nm})$ light via a microscope objective lens. When the nematodes recognized ultraviolet light in the region of the head (Figure $3 \mathrm{~A}-\mathrm{C}$ ), they reversed direction and moved away from the light region (Figure 3D). We measured the duration of this response and found that nematodes escaped from the light spot in 3.1, 4.1, 7.4, and 10 seconds when irradiated by ultraviolet, blue, blue-green, or green light, respectively (Figure 3E). These results indicate that nematodes avoid shorter wavelengths and that they are able to respond within a few seconds after light irradiation.

\section{Discussion}

Here, we found that $M$. incognita exhibits negative phototaxis. These nematodes efficiently responded to short-wavelength light. It has been reported that light stimuli elicits negative phototaxis in C. elegans, which lives in a dark environment like M. incognita (Ward et al., 2008). M. incognita may respond by returning to a dark environment like $C$. elegans when exposed to light. M. incognita individuals responded when their heads came under the light spot, indicating that they recognize light at the head. This negative phototactic behavior may be important for keeping the worms in a dark environment.

Ultraviolet light was the most effective for eliciting negative phototaxis in $M$. incognita. Ultraviolet light is thought to be more efficient at paralyzing worms than violet or blue light (Ward et al., 2008). The fruit fly, Drosophila melanogaster, has compound eye, and exhibits a negative phototaxis to ultraviolet light (Harris et al., 1976). Thus, it seems that the light avoidance response is generally essential for survival in insects.

Light signals are transduced by the activity of transient receptor potential (TRP) family channels in Drosophila (Wang and Montell, 2007). However, C. elegans and vertebrates utilize cyclic nucleotide-gate (CNG) channels (Fu and Yau, 2007; Ward et al., 2008). So, we searched homologues of C. elegans TAX-2 (NP_492427, NCBI) and the Drosophila TRP (NP_476768, NCBI) protein sequences using expressed sequence tag (EST) database of TBLASTN program (Altschul, 1997) at Meloidogyne genomic resources (http://meloidogyne.inra.fr/genomic_resources). Two ESTs of $M$. incognita, ra99h10 and rbd06 were identified to have significant homology with TAX-2, producing E-values of 1.5e-57 and 2e-36, respectively. However, no TRP homologs could be identified (data not shown). This suggests that $M$. incognita may have a mechanism similar to that of $C$. elegans for transducing light signals. Furthermore, M. incognita may utilize a group of ciliary sensory neurons for phototaxis, as is used in C. elegans (Ward et al., 2008). The two nematodes seem to have phototransduction cascades mediated by CNG channels, similar to those of vertebrates. It may be that the two soil-dwelling nematodes, $M$. incognita and C. elegans, have similar mechanisms for maintaining their position in the soil and that the nematodes inherited the CNG mechanism from their common ancestor. The CNG pathway may be an ancestral mechanism in the light-signaling pathway. In this case, urbilaterians, the ancestral group to vertebrates and insects (Adoutte et al., 1999), should have also possessed the CNG pathway.

\section{Acknowledgements}

We thank to Ngan Bui Thi for preparation of nematodes. We also thanks to Junko Hasegawa and Sachihiro Matsunaga for technical support to use Image J. S. Sawa, was supported by grants from KAKENHI (23119517, 24114009, 24370024, 24657035), Fuji foundation for protein research, and Asahi Glass Foundation.

\section{References}

Adoutte, A., Balavoine, G., Lartillot, N., \& De Rosa, R. (1999). Animal evolution. The end of the intermediate taxa? Trends Genet, 15, 104-108. http://dx.doi.org/10.1016/S0168-9525(98)01671-0

Altschul, S. F., Madden, T. L., Schaffer, A. A., Zhang, J., Zhang, Z., Miller, W., \& Lipman, D. J. (1997). Gapped BLAST and PSI-BLAST: A new generation of protein database search programs. Nucleic Acids Res., 25, 3389-3402. http://dx.doi.org/10.1093/nar/25.17.3389

Beron, D. M. (2007). Phototransduction in ganglion-cell photoreceptors. Pflugers Arch, 454, 849-855. 
Burr, A. H. (1979). Analysis of phototaxis in nematodes using directional statistics. J. Comp. Physiol., 134, 85-93.

Chitwood, B. G., \& Murphy, D. G. (1964). Observations on two marine monhysterids: their classification, cultivation, and behavior. Transactions of the American Microscopical Society, 83, 311-329. http://dx.doi.org/10.2307/3224742

Cho, S. W., Cho, J. H., Song, H. O., \& Park, C. S. (2005). Identification and characterization of a putative cyclic nucleotide-gated channel, CNG-1, in C. elegans. Mol. Cells, 19, 140-154.

Croll, N. A. (1966). The phototactic response and spectral sensitivity of Chromadorina viridis (Nematoda, Chromadorida) with a note on the nature of the paired pigment spots. Nematologica, 12, 610-614, http://dx.doi.org/10.1163/187529266X00464

Fu, Y., \& Yau, K. W. (2007). Phototransduction in mouse rods and cones. Pflugers Arch, 454, 805-819. http://dx.doi.org/10.1007/s00424-006-0194-y

Harris, W. A., Satrk, W. S., \& Walker, J. A. (1976). Genetic dissection of the photoreceptor system in the compound eye of Drosophila melanogaster. J. Physiol. (Lond.), 256, 415-439.

Kelber, A., Vorobyev, M., \& Osorio, D. (2003). Animal color vision-behavioral tests and physiological concepts. Biol. Rev. Camb. Philos. Soc, 78, 81-118. http://dx.doi.org/10.1017/S1464793102005985

Mclaren, D. J. (1973). Nematode sense organs. In B. Dawes (Ed.), Advances in Parasitology (pp. 195-265). London: Academic Press. http://dx.doi.org/10.1016/S0065-308X(08)60515-1

Nishiyama, H., Ngan, B. T., Nagasaki, S., Ejima, C., Ishida, T., \& Sawa, S. (2015). Protocol of root-knot nematode culture by hydroponic system and nematode inoculation to Arabidopsis. Nematological research, 45, 45-50. http://dx.doi.org/10.3725/jjn.45.45

Wang, T., \& Montell, C. (2007). Phototransduction and retinal degeneration in Drosophila. Pflügers Archiv-European Journal of Physiology, 454(5), 821-847. http://dx.doi.org/10.1007/s00424-007-0251-1

Ward, A., Liu, J., Feng, Z., \& Xu, X. S. (2008). Light-sensitive neurons and channels mediate phototaxis in $C$. elegans. Nature neuroscience, 11(8), 916-922. http://dx.doi.org/10.1038/nn.2155

\section{Copyrights}

Copyright for this article is retained by the author(s), with first publication rights granted to the journal.

This is an open-access article distributed under the terms and conditions of the Creative Commons Attribution license (http://creativecommons.org/licenses/by/4.0/). 